\title{
Intelligent Learning Objects: An Agent Approach to Create Interoperable Learning Objects
}

\author{
Ricardo Azambuja Silveira ${ }^{1}$, Eduardo Rodrigues Gomes ${ }^{2}$ and Rosa Vicari ${ }^{3}$ \\ 1 Universidade Federal de Santa Catarina, Campus Universitário, s/ $\mathrm{n}^{\circ}$ - \\ Caixa Postal 476 Florianópolis - SC, Brazil, silveira@inf.ufsc.br \\ 2 Universidade Federal do Rio Grande do Sul, Av. Bento Gonçalves, 9500 \\ - Campus do Vale - Bloco IV Porto Alegre - RS -Brazil, \\ ergomes@inf.ufrgs.br. \\ 3 Universidade Federal do Rio Grande do Sul, Av. Bento Gonçalves, 9500 \\ - Campus do Vale - Bloco IV Porto Alegre - RS - Brazil, rosa@inf.ufrgs.br
}

\begin{abstract}
Reusing learning material is very important to design learning environments for real-life learning. The reusability of learning objects results from the product of three main features: modularity, discoverability and interoperability. We proposed learning objects built based on agent architectures, called Intelligent Learning Objects (ILO). This paper discusses how the ILO approach can be used to improve the interoperability among learning objects, learning menagement systems (LMS) and pedagogical agents.
\end{abstract}

\section{Introduction}

This paper addresses the improvement of interoperability among learning objects in agent-based learning environments by integrating learning object technology and the multi-agent systems approach. According to Downes [3], Mohan \& Brooks [10], and Sosteric \& Hesemeier [11], a learning object is a piece of learning content that can be used several times in different courses or in different situations. According to Downes [3], the cost of developing learning materials for e-learning can be large, but if related courses are taught, the subject matter is similar and the cost of developing learning material can be shared. The learning object approach promises to reduce significantly the time and the cost required to develop e-learning courses. The use of reusable learning objects to create learning environments improves speed, flexibility and economy. 
A learning object must be modular, discoverable and interoperable in order to be reused [5]. To achieve these features and improve the efficiency, efficacy and reusability of learning objects, many people have dedicated a great effort. The main focus has been on standardization. Organizations such as the IMS Global Learning Consortium [7], IEEE [6], ARIADNE [1], and CanCore [2], have contributed significantly by defining indexing standards called metadata (data about data). Metadata contain the information to explain what the learning object is about, how to search, access, and identify it, and how to retrieve educational content according to a specific demand.

Mohan \& Brooks [10] point out some limitations of current learning objects. According to them an instructional designer must carefully examine a large amount of learning object. The task of finding the right object may be quite time consuming and the learning object metadata are not very useful in supporting pedagogical decisions.

On the other hand, the state of the art in intelligent learning environments (ILE) points to the use of architectures based on the multiagent paradigm [8]. Using the multiagent approach can result in faster, more versatile and lower cost systems. We proposed the intelligent learning object (ILO) approach as a useful way of enabling the reusability of agents making up pedagogical systems.

\section{Intelligent learning objects}

An intelligent learning object (ILO) is an agent that promotes learning experiences to students in the same way as learning objects used to do. For this reason, an ILO can be seen as a learning object built through the agent paradigm. The technological basis of this approach is combination of the technologies for learning objects and for multi-agent systems.

As a learning object, an intelligent learning object must be reusable. The reusability is given as a result of three features: interoperability, discoverability and modularity [5]. In a learning object approach, metadata is used to give discoverability by describing the pedagogical content of the learning object. To enable the discoverability feature in ILO, we have adopted the IEEE 1484.12.1 Standard for Learning Object Metadata [6]. The modularity of learning objects can be reached with a good pedagogical project. The design of the pedagogical task of an ILO must be made according to the expertise of some object matter specialists and pedagogical experts. Some interoperability can be reached by the use of wellknown standards. For this reason, we adopted two learning object standards: a) the IEEE 1484.12.1 Standard for Learning Object Metadata [6]; and, b) IEEE 1484.11.1 Standard for Learning Technology - Data Model for Content Object Communication. The 1484.11.1 standard is defined for communication of learning objects with Learning Management Systems (LMS). We use this standard in interactions among ILOs.

To assure interoperability among agents we have adopted the concepts defined by FIPA [4]. FIPA is an organization which defines standards to enable interoperability for multiagent systems. The main concern of FIPA is the definition of standards to enable the communication between agents. A well-defined 
communication framework is vital for interoperability among agents. We have used this reference model to define a communication framework for ILOs. The ILOs must use this framework in order to communicate with each other.

\section{The ILO multi-agent architecture}

The ILO architecture [12] encompasses three types of agents. The Intelligent Learning Object Agents are responsible for playing the role of learning objects. They generate learning experiences for the students in the same sense of learning objects. The LMS Agents are abstractions of learning management systems. They are responsible for dealing with the administrative and pedagogical tasks involving a learning environment as a whole, providing access to the ILOs for the students and storing information about the students.. The ILOR Agents are abstractions of learning object repository systems. They are responsible for storing data to permit a user or an agent to find ILOs. Figure 1 illustrates the proposed agent society.

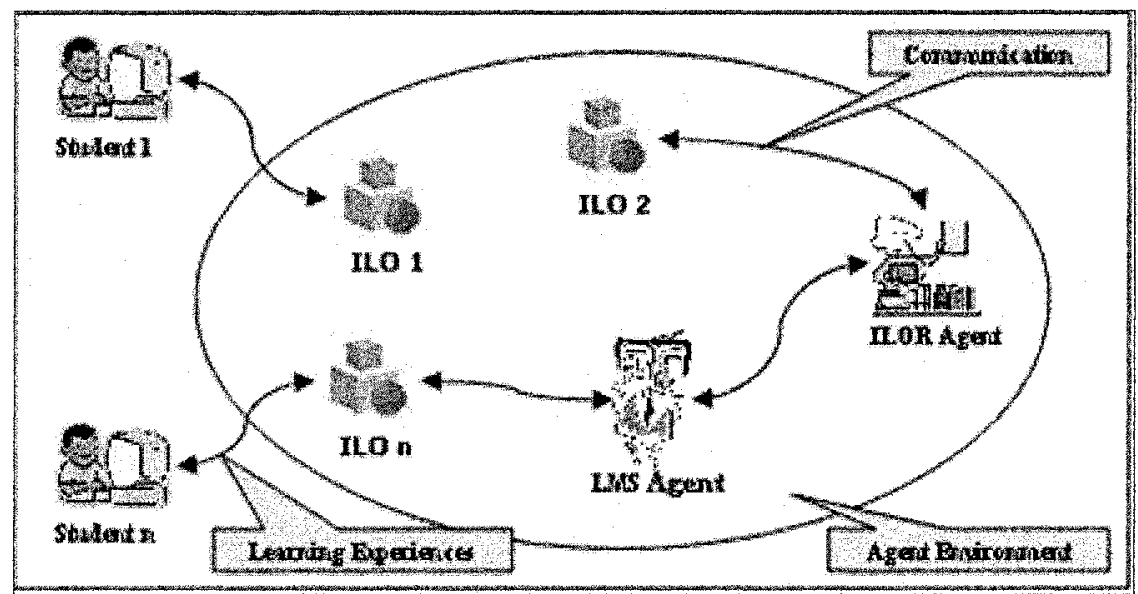

Figure 1. Proposed agent society

The students interact with the LMS agent in order to have learning experiences. The LMS agent asks the ILOR agent to search the appropriate ILO. The ILO is then responsible for generating learning experiences for the students. All these communications are performed by message exchanging using FIPA-ACL [4].

\section{Case study}

We developed an extended FIPA framework composed of a set of Java classes designed to build ILOs as easily as possible and applied this framework to the agentbased learning environment described by Lucas [9]. Such a system is composed of an ILO playing the role of a special calculator and an animated pedagogical agent 
(see Figure 2) playing the role of an animated tutor to help primary school students to learn some fundamental mathematical properties of multiplication.

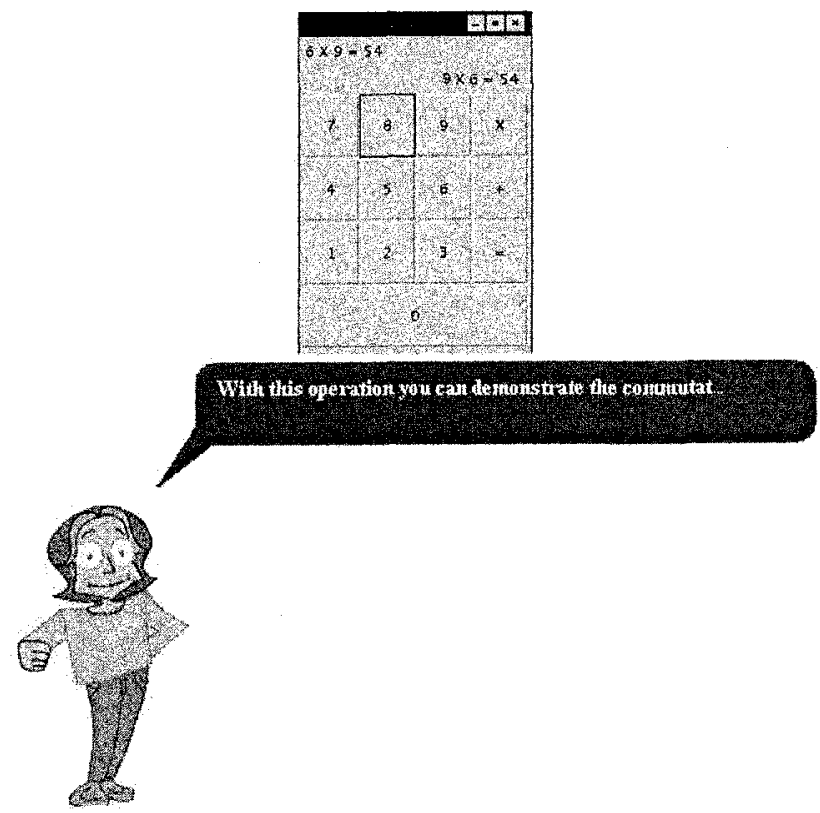

Figure 2. The APA giving an instruction.

The animated pedagogical agent works like an LMS and the calculator pedagogical agent is a typical ILO. The communication between the two agents is performed using the FIPA communication framework.

\section{Conclusions}

Intelligent learning objects are able to improve the adaptability, interoperability and interactivity of learning environments built with these kinds of components by the interaction among the learning objects and between learning objects and other agents. This concept of communication is more robust than a single method invocation as the object-oriented paradigm used to be.

The test bed application showed that the proposed approach of agent-based learning objects promises to be useful to improve the interoperability of learning objects, pedagogical agents and agent-based learning environments. The pedagogical agents are capable of providing suitable messages to the student and interact with the learning objects in a powerful way. This interaction provides more reality to the environment, and the student feels that he/she and the agents are active characters in the learning process.

While the development of educational content is converging to the use of standards towards reusability, we are still developing ad-hoc pedagogical-agentbased learning environments. This is the issue that this paper addressed. We should start to think about reusability when developing pedagogical agents. We need to go 
towards the use of reusable and interoperable pedagogical agents. The convergence between learning object and agent technologies seems to be promising.

\section{Acknowledgement}

This project is granted by Brazilian agencies: CNPq, CAPES and FAPERGS.

\section{References}

1. ARIADNE (2004). Alliance of remote instructional authoring \& distribution networks for Europe Retrieved July 7, 2005 http://ariadne.unil.ch.

2. CanCore (2004). Canadian Core About. Retrieved July 7, 2005 from http://www.cancore.ca/about.html.

3. Downes, S. (2001). Learning objects: resources for distance education worldwide. in International Review of Research in Open and Distance Learning, 2(1). 2001

4. FIPA: The foundation for Intelligent Physical Agents (2002). Specifications. retrieved July 7, 2005 from http://www.fipa.org.

5. Friesen, Norm (2001). What are Educational Objects? Interactive Learning Environments, 3 (9).

6. IEEE Learning Technology Standards Committee (2004). Specifications. retrieved July 7, 2005 from http://Itsc.ieee.org.

7. IMS Global Learning Consortium. (2004). Current specifications. retrieved July 7, 2005 from http://www.imsglobal org /specifications.cfm.

8. Johnson, W. Lewis; Shaw, Erin. (1997). Using agents to overcome deficiencies in webbased courseware. In: World Conference on Artificial Intelligence in Education, AI-ED, 8., 1997. Proceedings... retrieved July 7, 2005 from www.isi.edu/isd/johnson.html.

9. Lucas, J. P.; Widges, B.; Silveira, R. A. (2005). Inserting Animated Pedagogical Agents Inside Distributed Learning Environments by Means of FIPA Specifications. In: Fourth International Joint Conference on Autonomous Agents \& Multiagents Systems (AAMAS) - Proceedings of Agent-Based Systems for Human learning Workshop (ABSHL) 2005, Utrecht.

10. Mohan, P.and Brooks, C. (2003). Engineering a Future for Web-based Learning Objects. Proceedings of International Conference on Web Engineering, Oviedo, Asturias, Spain.

11. Sosteric, Mike, Hesmeier, Susan (2002). When is a Learning Object not an Object: A first step towards .a theory of learning objects. International Review of Research in Open and Distance Learning ISSN: 1492-3831

12. Silveira, R. A., Gomes, E. R, Vicari, R. M. (2005). Inteligent Learning Objects: An Agent-Based Approach of Learning Objects. In Weert, Tom Van, Tatnall, Arthur (Eds.) Information and Communication Technologies and Real-Life Learning.Boston Springer, $1103-110$. 